Supplementary information

\title{
A novel analytical approach for the simultaneous measurement of nitrate and DOC in soil water
}

Elad Yeshno ${ }^{1}$, Ofer Dahan ${ }^{1}$, Shoshana Bernstain ${ }^{1}$, Shlomi Arnon ${ }^{2}$

${ }^{1}$ Department of Hydrology \& Microbiology, Zuckerberg Institute for Water Research, Blaustein Institutes for Desert Research, Ben-Gurion University of the Negev, Israel

${ }^{2}$ Electrical and Computer Engineering Department, Ben-Gurion University of the Negev, Israel

Correspondence to: Elad Yeshno (Eladyes@post.bgu.ac.il) 


\section{Section S1: Selected agricultural sites}

The open crop field and citrus orchard sites are located next to the village of Nir Galim in Israel (3441 $13^{\prime \prime}$ E long; $31^{\circ} 49^{\circ} 42^{\prime \prime} \mathrm{N}$ Lat). Over the past 10 years, the crop field has been used for rainfed winter cereal and summer watermelon cultivation, fertilized mainly with dairy slurry manure. In 2015, the field was converted to irrigated jojoba cultivation. The citrus orchard is irrigated during the summer, in addition to the rainfall during the winter. Further information on these sites may be found at Turkeltaub et al., (2014). The additional study sites were two greenhouses used for rotating vegetable crops. One site practiced an organic regime that is based on the application of organic compost as the main fertilizer, while the other applied conventional fertigation methods. A detailed description of the VMS at each site was previously presented in Dahan et al., (2014); Turkeltaub et al., (2014, 2015a, 2016). Additional samples were taken from an open field located next to Kibbutz Afek (32 $50^{\prime} 14.7^{\prime \prime} \mathrm{N}$ $35^{\circ} 07^{\prime 28.6 " E) . ~}$ 


\section{Section S2: Chemical and spectral analyses of porewater samples}

Open field, inland: Database was obtained by analyzing water samples extracted from the soil, and was then used to form the nitrate calibration equation.

\begin{tabular}{|c|c|c|c|c|c|}
\hline \multicolumn{2}{|c|}{ Concentration (ppm) } & \multirow[b]{2}{*}{$\begin{array}{l}\text { Absorption at } \\
300 \mathrm{~nm} \text { (a.u.) }\end{array}$} & \multicolumn{2}{|c|}{ Concentration (ppm) } & \multirow[b]{2}{*}{$\begin{array}{c}\text { Absorption } \\
\text { at } 300 \mathrm{~nm} \\
\text { (a.u.) }\end{array}$} \\
\hline DOC & Nitrate & & DOC & Nitrate & \\
\hline 28.65 & 0.1 & 0.1409 & 3.58 & 0.01 & 0.0515 \\
\hline 28.09 & 196.18 & 0.1415 & 3.51 & 196.09 & 0.056 \\
\hline 27.29 & 476.29 & 0.1614 & 3.41 & 476.2 & 0.0768 \\
\hline 26.78 & 654.3 & 0.1763 & 3.35 & 654.22 & 0.0948 \\
\hline 26.05 & 909.18 & 0.2001 & 3.26 & 909.1 & 0.1158 \\
\hline 14.33 & 0.05 & 0.0841 & 1.79 & 0.01 & 0.0427 \\
\hline 14.04 & 196.13 & 0.0982 & 1.76 & 196.08 & 0.0483 \\
\hline 13.64 & 476.24 & 0.1202 & 1.71 & 476.2 & 0.0694 \\
\hline 13.39 & 654.25 & 0.1261 & 1.67 & 654.21 & 0.0837 \\
\hline 13.02 & 909.14 & 0.151 & 1.63 & 909.1 & 0.1074 \\
\hline 7.16 & 0.03 & 0.0552 & & & \\
\hline 7.02 & 196.1 & 0.071 & & & \\
\hline 6.82 & 476.21 & 0.0934 & & & \\
\hline 6.69 & 654.23 & 0.102 & & & \\
\hline 6.51 & 909.11 & 0.1219 & & & \\
\hline
\end{tabular}

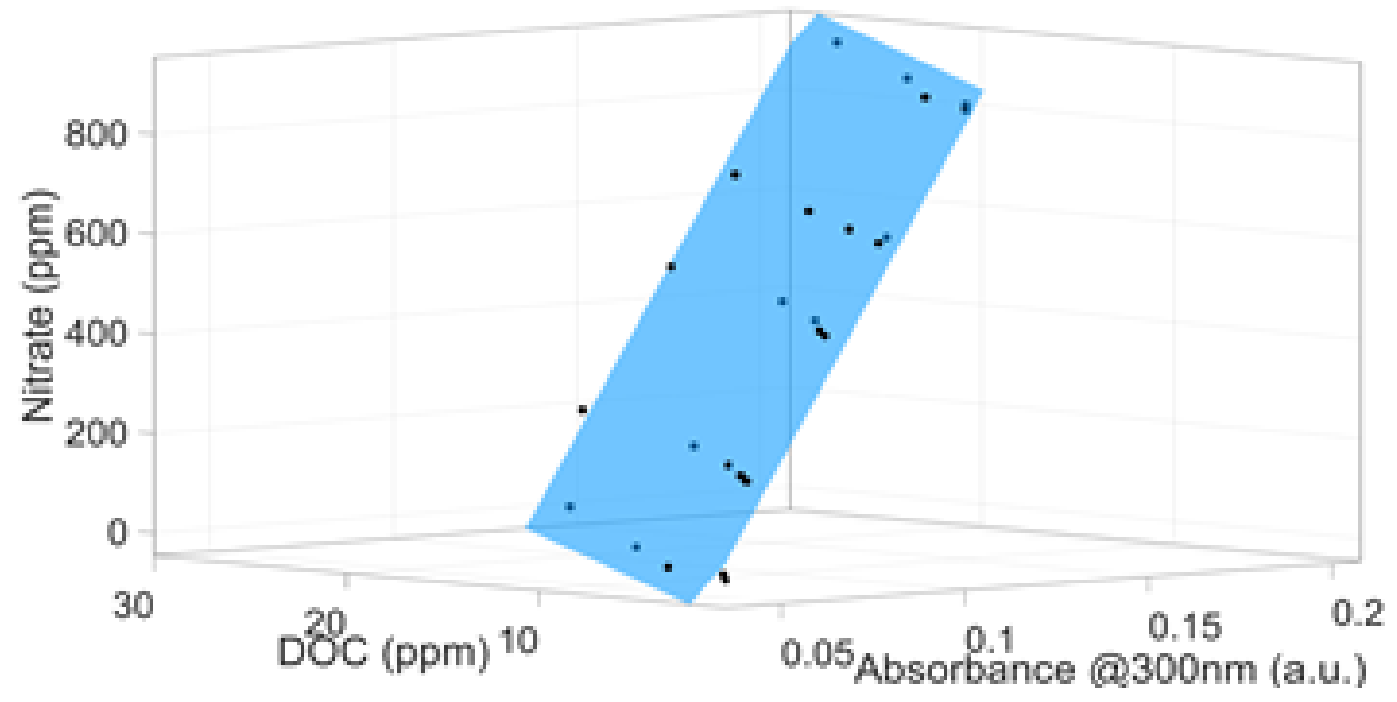


Organic greenhouse: Database was obtained by analyzing water samples extracted from the soil, and was then used to form the nitrate calibration equation.

\begin{tabular}{|c|c|c|c|c|c|}
\hline \multicolumn{2}{|c|}{ Concentration (ppm) } & \multirow[b]{2}{*}{$\begin{array}{l}\text { Absorption at } \\
300 \mathrm{~nm} \text { (a.u.) }\end{array}$} & \multicolumn{2}{|c|}{ Concentration (ppm) } & \multirow[b]{2}{*}{$\begin{array}{c}\text { Absorption } \\
\text { at } 300 \mathrm{~nm} \\
\text { (a.u.) }\end{array}$} \\
\hline DOC & Nitrate & & DOC & Nitrate & \\
\hline 12.50 & 2.63 & 0.14 & 36.06 & 46.03 & 0.33 \\
\hline 12.02 & 40.99 & 0.14 & 34.72 & 81.37 & 0.32 \\
\hline 11.57 & 76.50 & 0.14 & 31.25 & 173.23 & 0.30 \\
\hline 10.42 & 168.85 & 0.14 & 26.79 & 291.34 & 0.28 \\
\hline 8.93 & 287.59 & 0.13 & 20.83 & 448.82 & 0.26 \\
\hline 6.94 & 445.90 & 0.14 & 14.42 & 618.41 & 0.22 \\
\hline 4.81 & 616.39 & 0.13 & 50.00 & 10.50 & 0.45 \\
\hline 25.00 & 5.25 & 0.24 & 48.08 & 48.56 & 0.43 \\
\hline 24.04 & 43.51 & 0.23 & 46.30 & 83.80 & 0.41 \\
\hline 23.15 & 78.94 & 0.23 & 41.67 & 175.42 & 0.40 \\
\hline 20.83 & 171.04 & 0.22 & 35.71 & 293.21 & 0.36 \\
\hline 17.86 & 289.46 & 0.21 & 27.78 & 450.28 & 0.31 \\
\hline 13.89 & 447.36 & 0.20 & 19.23 & 619.42 & 0.28 \\
\hline 9.62 & 617.40 & 0.17 & & & \\
\hline 37.50 & 7.88 & 0.33 & & & \\
\hline
\end{tabular}

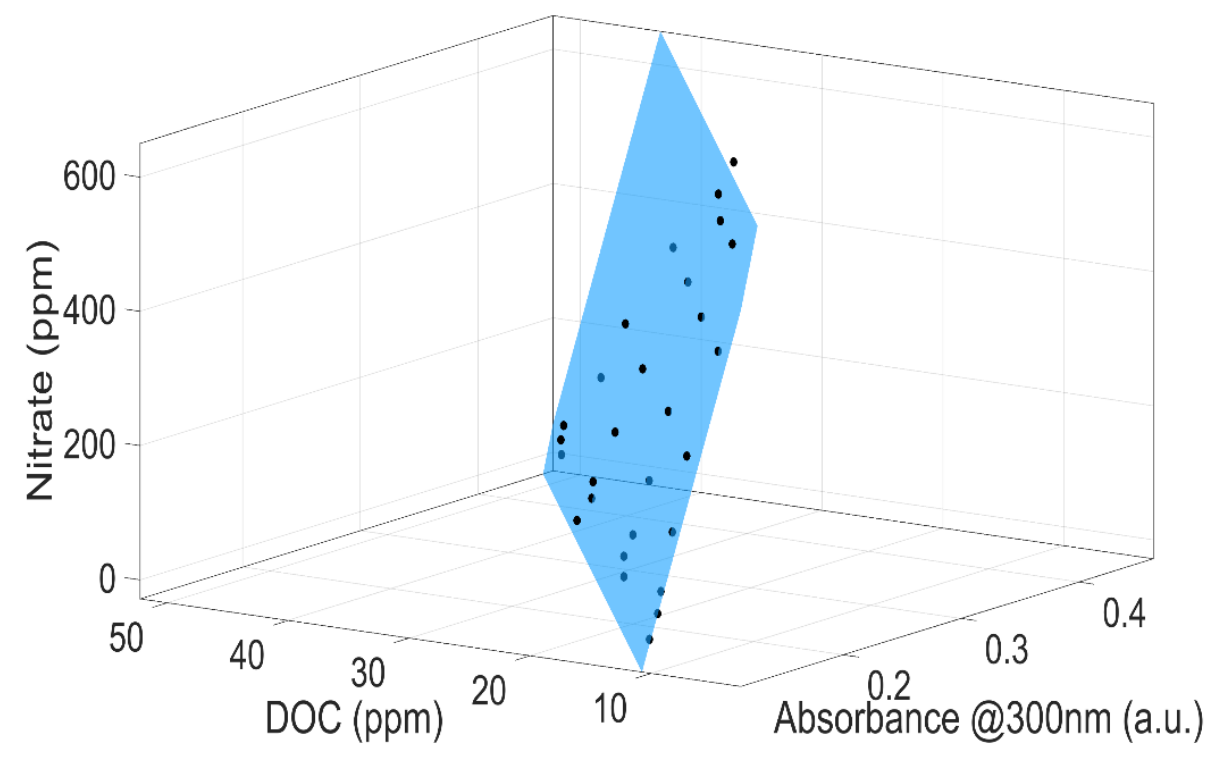


Open crop field, coastal plain: Database was obtained by analyzing water samples extracted from the soil, and was then used to form the nitrate calibration equation.

\begin{tabular}{|c|c|c|c|c|c|}
\hline \multicolumn{2}{|c|}{ Concentration (ppm) } & \multirow[b]{2}{*}{$\begin{array}{l}\text { Absorption at } \\
300 \mathrm{~nm} \text { (a.u.) }\end{array}$} & \multicolumn{2}{|c|}{ Concentration (ppm) } & \multirow[b]{2}{*}{$\begin{array}{c}\text { Absorption } \\
\text { at } 300 \mathrm{~nm} \\
\text { (a.u.) }\end{array}$} \\
\hline DOC & Nitrate & & DOC & Nitrate & \\
\hline 12.50 & 81.70 & 1.26 & 7.46 & 10.21 & 0.18 \\
\hline 12.02 & 276.18 & 1.25 & 7.32 & 206.09 & 0.20 \\
\hline 11.57 & 554.00 & 1.23 & 7.11 & 485.92 & 0.22 \\
\hline 10.42 & 730.56 & 1.23 & 6.97 & 663.75 & 0.23 \\
\hline 8.93 & 983.36 & 1.22 & 6.78 & 918.38 & 0.25 \\
\hline 6.94 & 40.85 & 0.66 & 3.73 & 5.11 & 0.11 \\
\hline 4.81 & 236.13 & 0.68 & 3.66 & 201.08 & 0.12 \\
\hline 25.00 & 515.10 & 0.66 & 3.55 & 481.05 & 0.14 \\
\hline 24.04 & 692.38 & 0.66 & 3.49 & 658.98 & 0.16 \\
\hline 23.15 & 946.23 & 0.66 & 3.39 & 913.73 & 0.18 \\
\hline 20.83 & 20.43 & 0.34 & & & \\
\hline 17.86 & 216.10 & 0.35 & & & \\
\hline 13.89 & 495.64 & 0.38 & & & \\
\hline 9.62 & 673.29 & 0.37 & & & \\
\hline 37.50 & 927.66 & 0.40 & & & \\
\hline
\end{tabular}

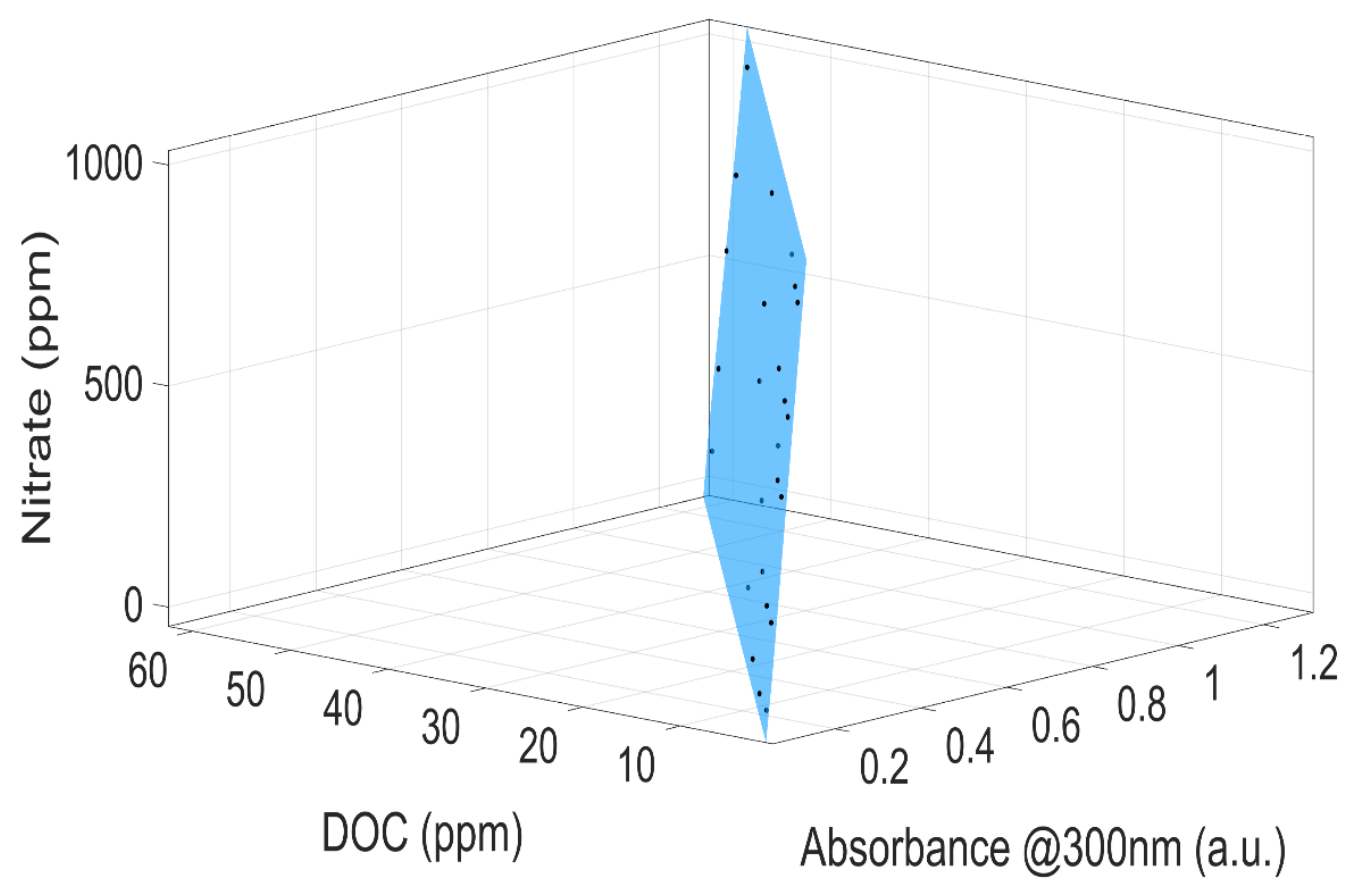


Citrus orchard: Database obtained by analyzing water samples extracted from the soil, and was then

\begin{tabular}{|c|c|c|c|c|c|}
\hline \multicolumn{2}{|c|}{ Concentration (ppm) } & \multirow[b]{2}{*}{$\begin{array}{l}\text { Absorption at } \\
300 \mathrm{~nm} \text { (a.u.) }\end{array}$} & \multicolumn{2}{|c|}{ Concentration (ppm) } & \multirow[b]{2}{*}{$\begin{array}{c}\text { Absorption } \\
\text { at } 300 \mathrm{~nm} \\
\text { (a.u.) }\end{array}$} \\
\hline DOC & Nitrate & & DOC & Nitrate & \\
\hline 24.95 & 2.02 & 0.09 & 83.17 & 173.39 & 0.24 \\
\hline 49.90 & 4.04 & 0.15 & 17.82 & 287.16 & 0.10 \\
\hline 74.85 & 6.05 & 0.19 & 35.64 & 288.60 & 0.14 \\
\hline 99.80 & 8.07 & 0.26 & 53.46 & 290.04 & 0.18 \\
\hline 23.99 & 40.40 & 0.09 & 71.29 & 291.48 & 0.22 \\
\hline 47.98 & 42.34 & 0.14 & 13.86 & 445.57 & 0.12 \\
\hline 71.97 & 44.28 & 0.28 & 27.72 & 446.69 & 0.15 \\
\hline 95.96 & 46.22 & 0.26 & 41.58 & 447.81 & 0.17 \\
\hline 23.10 & 75.94 & 0.11 & 55.44 & 448.93 & 0.21 \\
\hline 46.20 & 77.81 & 0.14 & 9.60 & 616.16 & 0.12 \\
\hline 69.31 & 79.68 & 0.19 & 19.19 & 616.94 & 0.14 \\
\hline 92.41 & 81.55 & 0.25 & 28.79 & 617.71 & 0.16 \\
\hline 20.79 & 168.35 & 0.10 & 38.38 & 618.49 & 0.18 \\
\hline 41.58 & 170.03 & 0.18 & & & \\
\hline 62.38 & 171.71 & 0.19 & & & \\
\hline
\end{tabular}

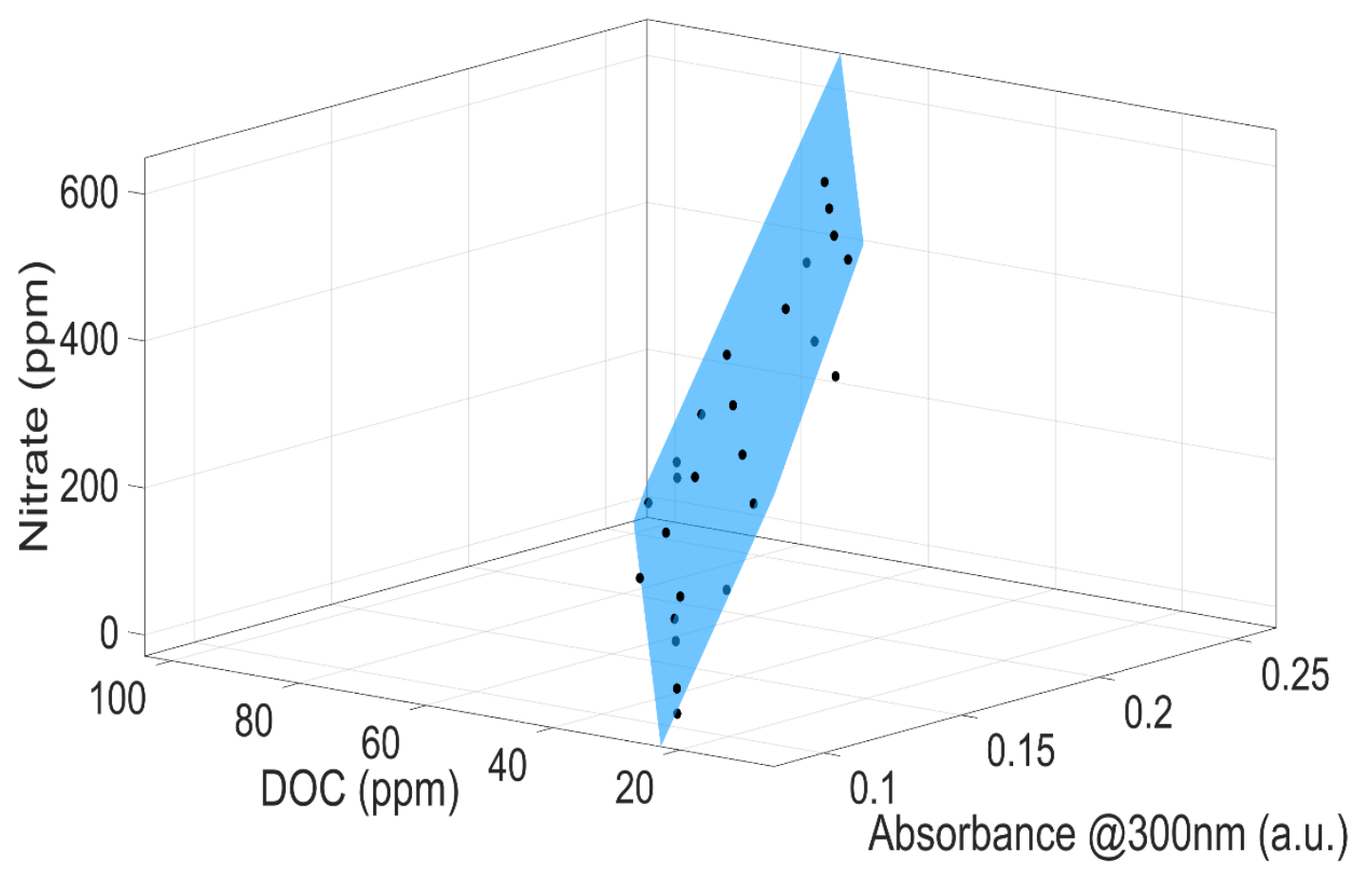


Hummus and soil mixture extract: Database was obtained by analyzing water samples extracted from the soil, and was then used to form the nitrate calibration equation.

\begin{tabular}{|c|c|c|c|c|c|}
\hline \multicolumn{2}{|c|}{ Concentration (ppm) } & \multirow[b]{2}{*}{$\begin{array}{l}\text { Absorption at } \\
300 \mathrm{~nm} \text { (a.u.) }\end{array}$} & \multicolumn{2}{|c|}{ Concentration (ppm) } & \multirow[b]{2}{*}{$\begin{array}{l}\text { Absorption } \\
\text { at } 300 \mathrm{~nm} \\
\text { (a.u.) }\end{array}$} \\
\hline DOC & Nitrate & & DOC & Nitrate & \\
\hline 7.2 & 18.53 & 0.1115 & 14.9 & 88.16 & 0.2039 \\
\hline 7.1 & 68.19 & 0.1185 & 14.7 & 185.82 & 0.2137 \\
\hline 7 & 166.04 & 0.1283 & 14.4 & 375.46 & 0.2205 \\
\hline 6.8 & 493.84 & 0.1505 & 14.2 & 512.96 & 0.2375 \\
\hline 10 & 25.74 & 0.1524 & 20 & 51.47 & 0.2669 \\
\hline 9.9 & 75.36 & 0.1461 & 19.9 & 100.97 & 0.2577 \\
\hline 9.8 & 173.14 & 0.16 & 19.7 & 198.49 & 0.2657 \\
\hline 9.6 & 363.03 & 0.175 & 19.3 & 387.9 & 0.2805 \\
\hline 9.5 & 500.7 & 0.181 & 19 & 525.21 & 0.2892 \\
\hline 12 & 30.88 & 0.1663 & & & \\
\hline 11.9 & 80.48 & 0.1722 & & & \\
\hline 11.8 & 178.21 & 0.176 & & & \\
\hline 11.6 & 368 & 0.1921 & & & \\
\hline 11.4 & 505.6 & 0.2015 & & & \\
\hline 15 & 38.6 & 0.2039 & & & \\
\hline
\end{tabular}

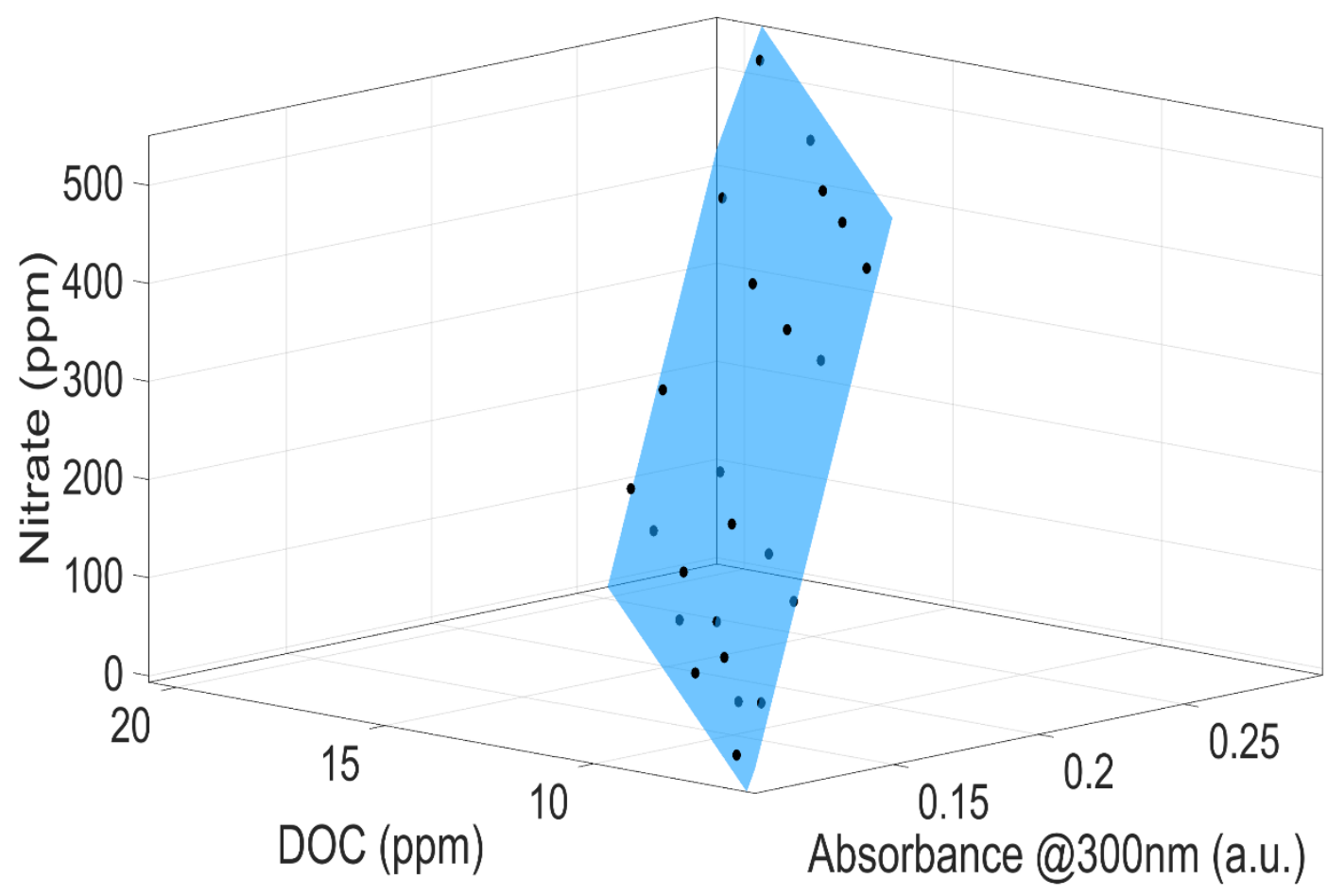




\section{References}

Dahan, O., A. Babad, N. Lazarovitch, E.E. Russak, and D. Kurtzman. 2014. Nitrate leaching from intensive organic farms to groundwater. Hydrol. Earth Syst. Sci. 18(1): 333-341. doi: 10.5194/hess-18-333-2014.

Dahan, O., R. Talby, Y. Yechieli, E. Adar, Y. Enzel, N. Lazarovitch, Y. Enzel, N. Lazarovitch, and Y. Enzel. 2009. In Situ Monitoring of Water Percolation and Solute Transport Using a Vadose Zone Monitoring System. Vadose Zo. J. 8(4): 916. doi: 10.2136/vzj2008.0134.

Rimon, Y., O. Dahan, R. Nativ, and S. Geyer. 2007. Water percolation through the deep vadose zone and groundwater recharge: Preliminary results based on a new vadose zone monitoring system. Water Resour. Res. 43(5): 1-12. doi: 10.1029/2006WR004855.

Rimon, Y., R. Nativ, and O. Dahan. 2011. Physical and Chemical Evidence for Pore-Scale DualDomain Flow in the Vadose Zone. Vadose Zo. J. 10(1): 322-331. doi: 10.2136/vzj2009.0113.

Turkeltaub, T., O. Dahan, and D. Kurtzman. 2014. Investigation of Groundwater Recharge under Agricultural Fields Using Transient Deep Vadose Zone Data. Vadose Zo. J. 13(4). doi: 10.2136/vzj2013.10.0176.

Turkeltaub, T., D. Kurtzman, and O. Dahan. 2016. Real-time monitoring of nitrate transport in deep vadose zone under a crop field - implications for groundwater protection. Hydrol. Earth Syst. Sci. Discuss. (February): 1-31. doi: 10.5194/hess-2016-63.

Turkeltaub, T., D. Kurtzman, E.E. Russak, and O. Dahan. 2015. Impact of switching crop type on water and solute fluxes in deep vadose zone. Water Resour. Res. 51(3): 9828- 9842. doi: 10.1002/2015WR017612. 